\title{
UNIQUENESS OF CONTROL SETS FOR PERTURBATIONS OF LINEAR SYSTEMS
}

\author{
FRITZ COLONIUS AND MARCO SPADINI
}

\begin{abstract}
Linear systems with controllable $(A, B)$ and bounded control range have a unique control set. This control set is bounded if and only if $A$ is hyperbolic. Then uniqueness also remains valid under small nonlinear perturbations. Examples show that for nonhyperbolic $A$ small nonlinear perturbations may lead to infinitely many (invariant) control sets.
\end{abstract}

\section{INTRODUCTION}

In this paper, we study the control sets for small nonlinear perturbations of linear control processes. More precisely we consider the maximal subsets of the state space $\mathbf{R}^{d}$ where complete controllability of the following perturbation of a linear control process (with restricted controls) holds

$$
\dot{x}(t)=A x(t)+B u(t)+\varepsilon F(u(t), x(t), \varepsilon), \quad u(t) \in U,
$$

where $U$ is a compact and convex subset of $\mathbf{R}^{m}$ with nonvoid interior, and $A$ and $B$ are constant matrices of respective dimensions $d \times d$ and $m \times d$. We assume that the pair $(A, B)$ is controllable, i.e., $\operatorname{rank}\left[B, A B, \ldots, A^{d-1} B\right]=d$, and that $F$ is a $C^{1}$-function. We also assume that

$$
\left\|D_{1} F\right\| \leq M_{1} \text { and }\left\|D_{2} F\right\| \leq M_{2} \text { uniformly. }
$$

Throughout we assume that for all $x_{0} \in \mathbf{R}^{d}$ and all controls $u$ there exists a unique solution $\varphi\left(t, x_{0}, u\right), t \in \mathbf{R}$, of (1.1) with initial value $\varphi\left(0, x_{0}, u\right)=x_{0}$.

The term $u(t)$ may be interpreted as a control function or as a time varying perturbation acting on the system. Control sets are of interest, in particular, since they contain all limit sets of the trajectories as time tends to infinity. Furthermore, they are related to the support of invariant measures for associated stochastic systems, compare [4]. This paper is focused on control sets with nonempty interior, as it is known that control sets which do not enjoy this property may have a very complicated structure (see e.g. [3] for examples). It is known, that the unperturbed equation (with $\varepsilon=0$ ) has a unique control set (with nonempty interior), if the pair $(A, B)$ is controllable and $0 \in \operatorname{int} U$. As shown by simple examples (see Section 2 , below), the number of the control sets of (1.1) may vary dramatically when $\varepsilon$ changes from zero to non zero values.

The main aim of this paper is to give conditions ensuring the existence of exactly one control set with nonvoid interior when $\varepsilon$ is small enough. It will turn out that hyperbolicity of the matrix $A$ is the crucial assumption.

1991 Mathematics Subject Classification. 93B05 ; 93C10 .

Key words and phrases. Control sets, hyperbolicity, perturbed systems.

M. Spadini was supported by the Nonlinear Control Network, TMR program. 
As an application, we consider the following control process:

$$
\dot{x}(t)=A x(t)+B u(t)+G(u(t), x(t)), \quad u(t) \in U,
$$

where $U$ is compact and convex in $\mathbf{R}^{m}$ and $G: \mathbf{R}^{m} \times \mathbf{R}^{d} \rightarrow \mathbf{R}^{d}$ is $C^{1}$, and we prove that if there exist $M_{1}>0$ and $M_{2}>0$ (depending only on $A$ and $B$ ) such that, for any $G$ which satisfies $\left\|D_{1} G\right\| \leq M_{1}$ and $\left\|D_{2} G\right\| \leq M_{2}$ uniformly, (1.3) admits exactly one control set with nonvoid interior. Further applications will be shown in a forthcoming paper.

In Section 2, we recall the definition of control sets and give conditions which imply that in the interior of a control set there exists a periodic trajectory corresponding to a continuous control. Then we give a number of examples which show that (1.1) may admit multiple control sets for any $\varepsilon>0$, while it has a unique control set for $\varepsilon=0$. In Section 3 we discuss properties of the unique control set for the linear system. In particular, we give conditions ensuring its boundedness. In Section 4, the nonlinear problem is discussed.

Notation. We denote by $C_{T}\left(\mathbf{R}^{d}\right), T>0$, the space of continuous $T$-periodic function $y: \mathbf{R} \rightarrow \mathbf{R}^{d}$ endowed with the sup-norm $\|y\|_{0}:=\max \{|y(t)|, t \in[0, T]\}$. Similarly, $C_{T}^{1}\left(\mathbf{R}^{d}\right)$ is the space of $T$-periodic continuously differentiable functions $y: \mathbf{R} \rightarrow \mathbf{R}^{d}$ endowed with the norm $\|y\|_{1}:=\max \left\{\|y\|_{0},\|\dot{y}\|_{0}\right\}$.

\section{Problem Formulation And Examples}

In this section, we give some definitions and prove preliminary results on control sets. Then some examples and counterexamples are discussed.

Consider the system

$$
\dot{x}(t)=f(x(t), u(t)), \quad u(t) \in U,
$$

where $U \subset \mathbf{R}^{m}$ is bounded and $f$ is $C^{1}$. We assume that unique solutions $\varphi\left(t, x_{0}, u\right)$, $t \in \mathbf{R}$, exist for all $x_{0} \in \mathbf{R}^{d}$ and all measurable control functions $u$. A useful notion that we use in the sequel is local accessibility, i.e. the system (2.1) is locally accessible if, for all $T>0$ and $x$

$$
\operatorname{int}\{\varphi(t, x, u), T \geq t>0 \text { and } u: \mathbf{R} \rightarrow U, \text { piecewise continuous }\} \neq \emptyset .
$$

In the sequel, we show that for $\varepsilon>0$ small enough one always has the local accessibility of (1.1) (see Remark 4.4). We start with the following definition.

Definition 2.1. A subset $D$ of $\mathbf{R}^{d}$ with nonvoid interior is a control set of (2.1) if for all $x \in D$ one has

$$
D \subset \operatorname{cl}\{\varphi(t, x, u), t>0 \text { and } u: \mathbf{R} \rightarrow U, \text { piecewise continuous }\},
$$

and $D$ is a maximal subset of $\mathbf{R}^{d}$ with this property.

This definition does not change if piecewise continuous controls are replaced by locally integrable ones (cp. [3], Section 3.2). If local accessibility is assumed, exact controllability in the interior of control sets holds. Thus for all $x, y \in \operatorname{int} D$ there are $T>0$ and a piecewise continuous control $u$ such that $\varphi(T, x, u)=y$. However, in the next section we will need this property for a continuous control function. We can guarantee this under a controllability condition for the linearized system. 
Proposition 2.2. Let $D$ be a control for (2.1) set with nonvoid interior, and assume that local accessibility holds in D. Suppose that there is a point $x_{0} \in \operatorname{int} D$, for which there are a constant control $u_{0} \in \operatorname{int} U$ and a time $T_{0}>0$ such that the linearized control system

$$
\dot{y}=D_{1} f\left(\varphi\left(t, x_{0}, u_{0}\right), u_{0}\right) y+D_{2} f\left(\varphi\left(t, x_{0}, u_{0}\right), u_{0}\right) u(t), \quad u(t) \in \mathbf{R}^{m},
$$

is controllable on every interval $[0, T], T_{0} \geq T>0$.

Then there are $T_{1}>0$ and a continuous control function $u_{1} \in \mathcal{U}$ such that $\left(\varphi\left(\cdot, x_{0}, u_{1}\right), u_{1}\right)$ is $T_{1}$-periodic.

Proof. As in [7], Section 3.7, Th. 7, the map

$$
\alpha: L_{\infty}\left([0, T], \mathbf{R}^{m}\right) \rightarrow \mathbf{R}^{d}, \quad u \mapsto \varphi\left(T, x_{0}, u\right)
$$

is continuously differentiable. By the controllability assumption, it follows that the restriction

$$
\alpha:\left\{u \in C\left([0, T], \mathbf{R}^{m}\right), u(0)=u(T)=u_{0}\right\} \rightarrow \mathbf{R}^{d}, \quad u \mapsto \varphi\left(T, x_{0}, u\right),
$$

has a surjective derivative at $u(t) \equiv u_{0} \in \operatorname{int} U$ (this can be derived from [7] sec. 2.8, Th. 1). Hence, by the Surjective Mapping Theorem (see e.g. [5]), the set

$$
Q:=\left\{\begin{array}{ll}
y \in \mathbf{R}^{d}, & \text { there is a continuous control } u \in \mathcal{U} \text { with } \\
u(0)=u(T)=u_{0} \text { and } y=\varphi\left(T, x_{0}, u\right)
\end{array}\right\}
$$

has nonvoid interior. Without loss of generality, we may assume that $T>0$ is small enough such that $Q \subset$ int $D$.

Pick $y \in \operatorname{int} Q$. By the local accessibility assumption, controllability in the interior of $D$ holds. Hence one finds a (piecewise constant) control $v$ and $S>0$ with $\varphi(S, y, v)=x_{0}$. Since the final value problem depends continuously on the right hand side, one also finds a continuous control $w \in \mathcal{U}$ with $w(0)=w(S)=u_{0}$ and $z \in \operatorname{int} Q$ with $\varphi(S, z, w)=x_{0}$. By the definition of $Q$ there is a continuous control $u \in \mathcal{U}$ with $u(0)=u(T)=u_{0}$ and $\varphi\left(T, x_{0}, u\right)=z$.

Concatenation of $v$ and $w$ and periodic continuation yields a continuous $(T+S)$ periodic control $u_{1}$ with $\varphi\left(T+S, x_{0}, u_{1}\right)=x_{0}$. With $T_{1}:=T+S$, the corresponding trajectory is $T_{1}$-periodic .

We note the following consequence for a control system of the form (1.1).

Proposition 2.3. Consider the control system (1.1), assume that $(A, B)$ is controllable and that $U$ is bounded. Then there exists a constant $c_{*}$ depending only on A, $B, M_{1}, M_{2}$ (an explicit expression for $c_{*}$ will be given in Remark 4.4) such that for all $\varepsilon \in\left(0, c_{*}\right]$, and every control set $D$ with nonvoid interior the following holds. For every $x_{0} \in \operatorname{int} D$ there are $T>0$ and a continuous control function $u_{0} \in \mathcal{U}$ such that $\left(\varphi\left(\cdot, x_{0}, u_{0}\right), u_{0}\right)$ is T-periodic.

Proof. The controllability assumption together with the boundedness of the derivative of $F$ implies that there exists a constant $c_{*}>0$ depending only on $A, B$, $M_{1}$ and $M_{2}$ such that, for $\varepsilon \in\left(0, c_{*}\right]$, and $u_{0} \in \operatorname{int} U$ the linearized system is controllable on arbitrarily short time intervals. Hence the assertion follows from the preceding proposition. 
Next we turn to a number of examples which illustrate the behavior of control sets for $\varepsilon=0$ and $\varepsilon>0$. They show that the unique control set of the unperturbed system may split into different control sets for positive $\varepsilon$.

Example 2.4. Let

$$
F(x, \varepsilon)= \begin{cases}\frac{1-\cos (\varepsilon \pi x)}{\varepsilon} & \text { for } x \in[-2 / \varepsilon, 2 / \varepsilon] \text { and } \varepsilon \neq 0 \\ 0 & \text { otherwise }\end{cases}
$$

and consider the scalar control process $\dot{x}(t)=u(t)+\varepsilon F(x(t), \varepsilon)$ with $u(t) \in[-1,1]$. The linear system $\dot{x}(t)=u$ is obviously controllable and, while for $\varepsilon=0$ the only control set is $\mathbf{R}$, for $\varepsilon>0$ there are 3 control sets, namely two unbounded intervals and one bounded interval.

In Example 2.4, $F$ depends explicitly on $\varepsilon$. The next example shows that things may go wrong even for $\varepsilon$-independent $F$ 's.

Example 2.5. For $n \in \mathbf{N} \cup\{0\}$, let

$$
F_{n}(x)=\left\{\begin{array}{ll}
n-n \cos \left(\frac{\pi\left(x-2^{n}\right)}{2^{n-1}}\right) & \text { for } x \in\left[2^{n}, 2^{n+1}\right], \\
0 & \text { otherwise, }
\end{array} \quad F(x)=\sum_{n=1}^{\infty} F_{n}(x),\right.
$$

Consider the scalar control process $\dot{x}(t)=u(t)+\varepsilon F(x(t))$ with $u(t) \in[-1,1]$. Then, for $\varepsilon=0$ the only control set is $\mathbf{R}$; whereas, for $\varepsilon>0$ there are infinitely many control sets with nonempty interior.

In both Examples 2.4 and 2.5, the matrix $A$ is singular. Below, we exhibit an example with nonsingular $A$, where multiple birth of control sets does arise. To do that, we have to increase the dimension by one.

Example 2.6. Consider the control process (1.1) with

$$
A=\left(\begin{array}{cc}
0 & 1 \\
-1 & 0
\end{array}\right), \quad B=\left(\begin{array}{ll}
1 & 0 \\
0 & 1
\end{array}\right)
$$

where $U$ is the closure of the unit ball in $\mathbf{R}^{2}$ with center at $(0,0)$, and

$$
F(x)=\rho\left(\sqrt{x^{2}+y^{2}}\right)(x, y), \text { with } \quad \rho(r)=\sum_{n=1}^{\infty} F_{n}(r)
$$

where $F_{n}$ is defined as in Example 2.5. With this choice of $A, B, F$ and $U$, for $\varepsilon=0$ there exists only one control set, and, although $(A, B)$ is controllable and $A$ is nonsingular, for $\varepsilon>0$ the control process (1.1) admits infinitely many control sets with nonempty interior.

Remark 2.7. Example 5.5 in [1] shows that for $\varepsilon \rightarrow 0^{+}$the number of control sets near an equilibrium of the system may tend to infinity.

Remark 2.8. The countably many control sets occurring for positive $\varepsilon$ in Example 2.5 are in fact invariant, i.e. they satisfy

$$
D=\operatorname{cl}\{\varphi(t, x, u): t>0, u: \mathbf{R} \rightarrow \text { Upiecewise continuous }\} .
$$

Hence this system has countably many different generic limit behaviours, see [2], [3] for precise statements. 
For the analysis of associated stochastic systems the invariant control sets are in 1-1 correspondence to the invariant measures (see [4] and [2]). Thus Example 2.5 gives a 'bifurcation' result for the associated stochastic systems.

\section{Periodic SOlutions of Linear Systems AND CONTROL SETS}

In this section we focus on the linear control process (with restricted controls) in $\mathbf{R}^{d}$ :

$$
\left\{\begin{array}{l}
\dot{x}(t)=A x(t)+B u(t) \\
u \in \mathcal{U}
\end{array}\right.
$$

where $A: \mathbf{R}^{d} \rightarrow \mathbf{R}^{d}$ and $B: \mathbf{R}^{m} \rightarrow \mathbf{R}^{d}$ are (constant) linear operators,

$$
\mathcal{U}=\{u: \mathbf{R} \rightarrow U \text { is continuous }\},
$$

and $U$ is a compact convex subset of $\mathbf{R}^{m}$. We will prove some results on the boundedness and uniqueness of control sets of (3.1). For related topics on linear control processes with restricted controls see e.g. [6], Sec. 5.3, or [7], Sec. 3.6. The results that will be presented go beyond their intrinsic interest as the underlying idea is at the root of the corresponding proofs for nonlinear perturbations provided in the next section.

Let us consider the periodic solutions of the following linear differential equation in $\mathbf{R}^{d}$

$$
\dot{x}=A x+y,
$$

where $A$ is a hyperbolic matrix and $y$ is a given periodic function. In particular, we will prove that there exists $K>0$ such that, if $y$ is $T$-periodic, then, for every $T>0$ given, the $T$-periodic solution $x$ of (3.2) (which is unique by the hyperbolicity of $A$ ) is bounded by a constant depending on $A$ and $\|y\|_{0}$.

Theorem 3.1. Let $A$ be hyperbolic (i.e. such that $\sigma(A) \cap i \mathbf{R}=\emptyset$ ). Then there exists $K>0$, depending only on $A$, such that for any $T>0$ and $y \in C_{T}\left(\mathbf{R}^{d}\right)$, the $T$-periodic solution $x$ of (3.2) satisfies $\|x\|_{1}<K\|y\|_{0}$.

The proof of this theorem relies on several lemmas discussing the behavior with respect to Jordan blocks.

Lemma 3.2. Let $a \neq 0$ and consider the scalar differential equation

$$
\dot{x}=a x+y \text {. }
$$

Then for every $T>0$ and $y \in C_{T}(\mathbf{R})$, there exists $K>0$ such that, if $x$ is the unique T-periodic solution of (3.3), then $\|x\|_{1}<K\|y\|_{0}$.

Proof. Assume first $a<0$. The unique $T$-periodic solution of (3.3) is given by

$$
x(t)=\frac{e^{t a}}{1-e^{T a}} \int_{0}^{T} e^{(T-s) a} y(s) d s+\int_{0}^{t} e^{(t-s) a} y(s) d s,
$$

hence

$$
|x(t)| \leq-\frac{2}{a}\|y\|_{0} .
$$

From (3.3) we find $|\dot{x}(t)| \leq|a|\|x\|_{0}+\|y\|_{0} \leq 2\|y\|_{0}$. And finally

$$
\|x\|_{1} \leq(2-2 / a)\|y\|_{0} .
$$


Assume now $a>0$ and consider the equation

$$
\dot{x}=-a x-\tilde{y}, \quad \tilde{y}(t):=-y(-t) .
$$

Any $T$-periodic solution of this last equation is a time reversed $T$-periodic solution of (3.3). Hence the assertion follows from the first part of the proof.

Lemma 3.3. Assume that $A=\operatorname{diag}\left\{\alpha_{1}, \ldots, \alpha_{d}\right\}$ with $\alpha_{i} \in \mathbf{R} \backslash\{0\}$. Then for every $T>0$ and $y \in C_{T}\left(\mathbf{R}^{d}\right)$, there exists $K>0$ such that, if $x$ is the unique $T$-periodic solution of (3.2), then $\|x\|_{1}<K\|y\|_{0}$.

Proof. Let $y(t)=\left(y_{1}(t), \ldots, y_{d}(t)\right)$, equation (3.2) splits in the following system of $d$ uncoupled linear differential equations

$$
\left\{\begin{array}{c}
\dot{x}_{1}=\alpha_{1} x_{1}+y_{1}, \\
\vdots \\
\dot{x}_{d}=\alpha_{d} x_{d}+y_{d} .
\end{array}\right.
$$

The assertion follows applying Lemma 3.2 to each one of the equations above.

Lemma 3.4. Assume that the square $d \times d$ matrix $A$ has the following form:

$$
A=\left(\begin{array}{ccccc}
\alpha & 1 & 0 & \cdots & 0 \\
0 & \alpha & 1 & & \vdots \\
\vdots & & \ddots & \ddots & 0 \\
0 & \cdots & 0 & \alpha & 1 \\
0 & \ldots & \ldots & 0 & \alpha
\end{array}\right),
$$

with $\alpha \neq 0$. Then for every $T>0$ and $y \in C_{T}\left(\mathbf{R}^{d}\right)$, there exists $K>0$ such that, if $x$ is the unique T-periodic solution of (3.2), then $\|x\|_{1}<K\|y\|_{0}$.

Proof. With $y(t)=\left(y_{1}(t), \ldots, y_{d}(t)\right)$, the $d$-th component of equation (3.2) takes the form

$$
\dot{x}_{d}(t)=\alpha x_{d}(t)+y_{d}(t) .
$$

Hence by Lemma 3.2 we get that there exists $K_{d}>0$ such that

$$
\left\|x_{d}\right\|_{1} \leq K_{d}\left\|y_{d}\right\|_{0} \leq K_{d}\|y\|_{0}
$$

The $(d-1)$-st component of (3.2) has the form

$$
\dot{x}_{d-1}(t)=\alpha x_{d-1}(t)+x_{d}(t)+y_{d-1}(t) .
$$

Applying Lemma 3.2 again we get the existence of $K_{d-1}>0$ such that

$$
\left\|x_{d-1}\right\|_{1} \leq K_{d-1}\left\|x_{d}+y_{d-1}\right\|_{0} \leq\left(1+K_{d}\right) K_{d-1}\|y\|_{0} .
$$

Analogously we can then estimate $\left\|x_{d-2}\right\|_{1}$ and so on. Hence, in a finite number of steps, we get an estimate for every component of $x$.

Lemma 3.5. Let $a \neq 0$ and

$$
A=\left(\begin{array}{cc}
a & b \\
-b & a
\end{array}\right) .
$$

Then for every $T>0$ and $y \in C_{T}\left(\mathbf{R}^{2}\right)$, there exists $K>0$ such that, if $x$ is the unique T-periodic solution of (3.2), then $\|x\|_{1}<K\|y\|_{0}$. 
Proof. Consider the complex-valued differential equation

$$
\dot{z}=(a+i b) z+\eta,
$$

with $\eta(t)=y_{1}(t)+i y_{2}(t)$. Clearly it is enough to show that there exists a positive number $K$ such that for any $T$-periodic $y_{1}$ and $y_{2}$ the $T$-periodic solution $z$ of (3.4) satisfies

$$
|z| \leq K \sup _{t \in[0, T]}|\eta(t)| .
$$

Assume first $a<0$. As in the case of equation (3.3), the unique $T$-periodic solution of (3.4) is given by:

$$
z(t)=\frac{e^{t(a+i b)}}{1-e^{T(a+i b)}} \int_{0}^{T} e^{(T-s)(a+i b)} \eta(s) d s+\int_{0}^{t} e^{(t-s)(a+i b)} \eta(s) d s,
$$

Analogously to the proof of Lemma 3.2 and taking into account that $\left|e^{t(a+i b)}\right|=e^{t a}$ we get

$$
|z| \leq 2|a|^{-1} \sup _{t \in[0, T]}|\eta(t)| .
$$

In the case when $a>0$, the proof is performed, analogously to Lemma 3.2, by time reversal.

Lemma 3.6. For $a \neq 0$ define

$$
\mathcal{A}=\left(\begin{array}{cc}
a & b \\
-b & a
\end{array}\right), \quad I=\left(\begin{array}{ll}
1 & 0 \\
0 & 1
\end{array}\right)
$$

and let $A$ be the $(2 d \times 2 d)$-matrix given by

$$
A=\left(\begin{array}{cccc}
\mathcal{A} & I & & \\
& \mathcal{A} & \ddots & \\
& & \ddots & I \\
& & & \mathcal{A}
\end{array}\right) .
$$

Then for every $T>0$ and $y \in C_{T}\left(\mathbf{R}^{2 d}\right)$, there exists $K>0$ such that, if $x$ is the unique T-periodic solution of (3.2), then $\|x\|_{1}<K\|y\|_{0}$.

Proof. Let $y(t)=\left(y_{1}(t), \ldots, y_{2 d}(t)\right)$. Equation (3.2) splits into the following system of 2-dimensional differential equations

$$
\left\{\begin{array}{c}
\dot{\xi}_{1}=\mathcal{A} \xi_{1}+\xi_{2}+\eta_{1}, \\
\dot{\xi}_{2}=\mathcal{A} \xi_{2}+\xi_{3}+\eta_{2}, \\
\vdots \\
\dot{\xi}_{d}=\mathcal{A} \xi_{d}+\eta_{d}
\end{array}\right.
$$

where, for $j=1, \ldots, d$, we put $\eta_{j}=\left(y_{2 j-1}, y_{2 j}\right)$ and $\xi_{j}=\left(x_{2 j-1}, x_{2 j}\right)$.

Applying Lemma 3.5 to the $d$-th equation of the system above, we get the existence of a positive constant $K_{d}$ such that $\left\|\xi_{d}\right\|_{1}<K_{d}\left\|\eta_{d}\right\|_{0}$. Following the same argument of the proof of Lemma 3.4, we get an estimate of any component of $x$.

Proof of Theorem 3.1. Up to a coordinate change we can assume that the matrix $A$ is in real Jordan canonical form. Hence, equation (3.2) splits up into independent linear subsystems of the forms considered in Lemmas 3.3-3.6. 
Theorem 3.1 enables us to prove some facts about the control sets of (3.1). Defining $\mathcal{U}_{T}=\mathcal{U} \cap C_{T}\left(\mathbf{R}^{m}\right)$, we have that

$$
\operatorname{int} \mathcal{U}_{T}=\left\{u \in \mathcal{U}_{T}: u(t) \in \operatorname{int} U \text { for all } t \in \mathbf{R}\right\}
$$

We will need the following consequence of Proposition 2.3. Observe that local accessibility holds for (3.1) by the controllability assumption.

Lemma 3.7. Let $D$ be a control set of (3.1) with non empty interior and let $p$ belong to int $D$. Then there exists $u_{0} \in \operatorname{int} \mathcal{U}_{T}$ such that $\dot{x}(t)=A x(t)+B u_{0}(t)$ has a periodic orbit whose image contains $p$.

The remaining part of this section will be devoted to proving, as applications of Theorem 3.1, some facts about the boundedness of control sets with non empty interiors (Theorems 3.9 and 3.11, below), and a uniqueness result for such control sets (Theorem 3.12, below). These results are to be compared with those contained in [3], Chapter 3, where a uniqueness result for the control sets of a linear systems is proved assuming that $U$ contains the origin in its interior.

Notice that in our theorems below, we always assume the hyperbolicity of the matrix $A$. This assumption cannot be dropped since we are considering sets $U$ which do not necessarily contain the origin. In fact, for such $U$ 's we do not necessarily have the existence of control sets. The following simple example from [3] illustrates this fact:

Example 3.8. Consider the scalar control process

$$
\dot{x}(t)=u(t), \quad u(t) \in U \subset \mathbf{R} .
$$

If $U \subset(0, \infty)$ then there are no control sets at all. If $U=\{0\}$ then every point is a control set.

Theorem 3.9. Let $A$ be hyperbolic. Then the control sets of (3.1) which have non empty interior are bounded.

Proof. Assume by contradiction that it exists an unbounded control set with non empty interior. Then by Lemma 3.7, there exists an unbounded sequence of periodic solutions of (3.1). This contradicts Theorem 3.1 since $\sup _{u \in \mathcal{U}}\|u\|_{0} \leq \max \{|v|: v \in$ $U\}$ is finite.

We will also need the following

Lemma 3.10. Assume that the pair $(A, B)$ is controllable. Given $T>0$ and $\bar{u} \in \operatorname{int} \mathcal{U}_{T}$, every $T$-periodic solution of

$$
\dot{x}(t)=A x(t)+B \bar{u}(t),
$$

is contained in the interior of a control set of (3.1).

Proof. Denote by $\varphi(\cdot, u, p)$ the solution of the Cauchy problem

$$
\left\{\begin{array}{l}
\dot{x}(t)=A x(t)+B u(t), \\
x(0)=p,
\end{array}\right.
$$

and let $p_{0}$ be the starting point of a $T$-periodic solution of (3.5). Denote by $E_{T}$ the space of continuous functions $v$ such that $v(0)=v(T)=0$, and take

$$
\mathcal{V}=\left\{v \in E_{T}: \bar{u}(t)+v(t) \in \operatorname{int}(U)\right\} \text {. }
$$


Obviously $\mathcal{V}$ is an open subset of the Banach space $E_{T}$. Define $\Theta: \mathcal{V} \rightarrow \mathbf{R}^{m}$ as

$$
\Theta(v)=\varphi\left(T, \bar{u}+v, p_{0}\right)=e^{T A} p_{0}+\int_{0}^{T} e^{(T-s) A} B(\bar{u}(s)+v(s)) d s .
$$

Notice that $\Theta(0)=p_{0}$. For any $\omega \in E_{T}$, we have

$$
\Theta^{\prime}(0) \omega=\int_{0}^{T} e^{(T-s) A} B \omega(s) d s .
$$

The controllability assumption implies that $\Theta^{\prime}(0)$ is surjective. The Surjective Mapping Theorem (see e.g. [5]) implies that there exists a neighborhood $V_{0}$ of $p_{0}$ which is made up of images of $\Theta$. In particular, $p_{0}$ can be driven to any point of $V_{0}$.

Applying the same argument to the time reversed control process, we have that there exists a neighborhood $V_{1}$ of $p_{0}$, any point of which can be driven to $p_{0}$. Hence $V_{0} \cap V_{1}$ is contained in control set.

Take now any point $q \in \varphi\left([0, T], \bar{u}, p_{0}\right)$ and let $t_{0} \in[0, T]$ be such that $q=$ $\varphi\left(t_{0}, \bar{u}, p_{0}\right)$. By the continuity of $\varphi\left(t_{0}, \bar{u}, \cdot\right)$ there exists a neighborhood $W$ of $q$ such that

$$
\varphi\left(t_{0}, \bar{u}, \cdot\right)^{-1}(W) \subset V_{0} \cap V_{1} .
$$

Analogously, by the continuity of the time reversed system, shrinking $W$ if necessary, we can assume that

$$
\varphi\left(t_{0}, \bar{u}, W\right) \subset V_{0} \cap V_{1} .
$$

Hence, any point of $W$ can be driven to any other point of $W$. That is $W$ is contained in a control set. The assertion now follows from the compactness of $\varphi\left([0, T], \bar{u}, p_{0}\right)$.

This lemma can be used to prove two remarkable facts:

Theorem 3.11. Assume the pair $(A, B)$ is controllable and that $U$ is convex with $0 \in \operatorname{int}(U)$. Then the control sets of (3.1) which have nonempty interior are bounded if and only if $A$ is hyperbolic.

Proof. We already know that, if $A$ is hyperbolic, the control sets with nonempty interior are bounded.

Let $A$ be non hyperbolic. If $\operatorname{det} A=0$ then $\operatorname{ker} A \neq\{0\}$. Any point of $\operatorname{ker} A$ is a periodic solution of (3.1), which, by Lemma 3.10 is contained in the interior of a control set.

If $\operatorname{det} A \neq 0$ there exists a pair of conjugate imaginary eigenvalues, say $\pm i \beta$, $\beta \neq 0$. By the Jordan real canonical form of the matrix $A$, there exists a 2dimensional subspace $V$ of $\mathbf{R}^{m}$ such that

$$
\left.A\right|_{V}=\left(\begin{array}{cc}
0 & \beta \\
-\beta & 0
\end{array}\right)
$$

Hence each point of $V$ is the starting point of a periodic solution of $\dot{x}=A x$ (that is of equation (3.1) with control function $u(t) \equiv 0)$ and period $2 \pi / \beta$. Thus, by Lemma 3.10 each point of $V$ is contained in the interior of a control set.

Theorem 3.12. Assume that the pair $(A, B)$ is controllable, $A$ is hyperbolic and $U$ is convex. Then there exists a unique control set with non empty interior of (3.1). 
Proof. Let $T>0$ and $\bar{u} \in \operatorname{int} \mathcal{U}_{T}$. The hyperbolicity of $A$ guarantees the existence of a $T$-periodic solution of $\dot{x}(t)=A x(t)+B \bar{u}(t)$, whose image is, by Lemma 3.10, contained in the interior of a control set. This proves the existence part of the assertion.

Let us now prove the uniqueness. Assume by contradiction that there exist two control sets $D_{0}$ and $D_{1}$ with nonempty interior. By Lemma 3.7 we know that there exist periodic continuous controls $u_{0}$ and $u_{1}$ (say $T_{0^{-}}$and $T_{1}$-periodic, respectively) which take values in the interior of $U$ and such that the $T_{i}$-periodic solution of

$$
\dot{x}(t)=A x(t)+B u_{i}(t),
$$

is contained in $D_{i}, i=0,1$. Define

$$
u_{\lambda}(t)=\lambda u_{1}\left(\frac{T_{1}}{\lambda T_{1}+(1-\lambda) T_{0}} t\right)+(1-\lambda) u_{0}\left(\frac{T_{0}}{\lambda T_{1}+(1-\lambda) T_{0}} t\right) .
$$

Since $U$ is convex by assumption, one has $u_{\lambda} \in \operatorname{int} \mathcal{U}_{T_{\lambda}}$, with $T_{\lambda}=\lambda T_{1}+(1-\lambda) T_{0}$. The equation

$$
\dot{x}(t)=A x(t)+B u_{\lambda}(t),
$$

has a unique $T_{\lambda}$-periodic solution $x_{\lambda}(\cdot)$ whose image is contained in the interior of a control set by Lemma 3.10. We want to show that these images constitute a continuum joining $D_{0}$ and $D_{1}$. This will yield the desired contradiction.

Consider the time transformed system

$$
\dot{\xi}(\tau)=T_{\lambda}\left(A \xi(\tau)+B u_{\lambda}\left(\tau T_{\lambda}\right)\right),
$$

and observe that $\hat{x}(\lambda, \tau)=x_{\lambda}\left(T_{\lambda} \tau\right)$ gives its unique 1-periodic solution. Since the map $(\lambda, \tau) \mapsto \hat{x}(\lambda, \tau)$ is continuous, the set $\hat{x}([0,1] \times[0,1])$ is connected and coincides with the set of images of the maps $x_{\lambda}(\cdot)$, for $\lambda \in[0,1]$.

\section{Nonlinear PERTURBATIONS}

This section is devoted to studying the control process (1.1). The main result is Theorem 4.8 which states that, under reasonable assumptions, the uniqueness of control sets with nonvoid interior for (1.1) holds. As we mentioned the argument of the proof is inspired by that of Theorem 3.12 above, although the technical details are more subtle.

Consider the following nonlinear perturbation of a linear hyperbolic control system.

$$
\begin{aligned}
& \dot{\xi}(t)=A \xi(t)+B u(t)+\varepsilon F(u(t), \xi(t), \varepsilon), \\
& u(t) \in U .
\end{aligned}
$$

Throughout this section $F$ will be assumed $C^{1}$ with $\left\|D_{1} F(v, p, \varepsilon)\right\| \leq M_{1}$ and $\left\|D_{2} F(v, p, \varepsilon)\right\| \leq M_{2}$ uniformly. Furthermore, let $K_{A}$ denote the constant given by Theorem 3.1.

Lemma 4.1. Let $F$ and $A$ be as above. Take $\varepsilon \in\left[-\varepsilon_{0}, \varepsilon_{0}\right]$ with

$$
\varepsilon_{0}=\min \left\{1, \frac{1}{2 K_{A} M_{2}}\right\} \text {. }
$$

Then for every $T>0$, the differential equation (4.1a) has a unique T-periodic solution, $\xi(\cdot, u, \varepsilon)$, for $u \in \mathcal{U}_{T}$, and the map $\mathcal{U}_{T} \times\left[-\varepsilon_{0}, \varepsilon_{0}\right] \rightarrow C_{T}^{1}\left(\mathbf{R}^{m}\right)$ given by $(u, \varepsilon) \mapsto x(\cdot, u, \varepsilon)$ is continuous. 
Furthermore, assuming in addition that $U$ contains the origin of $\mathbf{R}^{m}$ in its interior, one has

$$
\sup _{t \in[0, T]}|x(t, u, \varepsilon)| \leq 2 c_{U} K_{A}\left(\|B\|+M_{1}\right), \quad c_{U}:=\max \{|v|: v \in U\},
$$

for every $u \in \mathcal{U}_{T}$ and $\varepsilon \in\left[-\varepsilon_{0}, \varepsilon_{0}\right]$.

Proof. Rewrite the equation (4.1a) in the form:

$$
L x-\bar{A} x-\bar{B} u-\varepsilon \bar{F}(u, x, \varepsilon)=0
$$

where we put

$$
\begin{array}{lll}
L: C_{T}^{1}\left(\mathbf{R}^{d}\right) \rightarrow C_{T}\left(\mathbf{R}^{d}\right) & \text { with } & (L x)(t)=\dot{x}(t), \\
\bar{A}: C_{T}^{1}\left(\mathbf{R}^{d}\right) \rightarrow C_{T}\left(\mathbf{R}^{d}\right) & \text { with } & (\bar{A} x)(t)=A x(t), \\
\bar{B}: C_{T}\left(\mathbf{R}^{m}\right) \rightarrow C_{T}\left(\mathbf{R}^{d}\right) & \text { with } & (\bar{B} u)(t)=B u(t) \\
\bar{F}: \mathcal{U}_{T} \times C_{T}^{1}\left(\mathbf{R}^{d}\right) \times \mathbf{R} \rightarrow C_{T}\left(\mathbf{R}^{d}\right) & \text { with } & \bar{F}(u, x, \varepsilon)(t)=F(u(t), x(t), \varepsilon)
\end{array}
$$

From Theorem 3.1 follows that there exists $K_{A}>0$ (independent of $T>0$ ) such that if $(L-\bar{A}) x=y$ then $\|x\|_{1} \leq K_{A}\|y\|_{0}$. In other words

$$
\left\|(L-\bar{A})^{-1}\right\| \leq K_{A} \text {. }
$$

Let $\Phi: \mathcal{U}_{T} \times C_{T}^{1}\left(\mathbf{R}^{d}\right) \times \mathbf{R} \rightarrow C_{T}^{1}\left(\mathbf{R}^{d}\right)$, be given by

$$
\Phi(u, x, \varepsilon)=-(L-\bar{A})^{-1}(\bar{B} u+\varepsilon \bar{F}(u, x, \varepsilon)) .
$$

Then equation (4.3) is equivalent to

$$
\Phi(u, x, \varepsilon)=x .
$$

Let us show that for $|\varepsilon|<\left(2 K_{A} M_{2}\right)^{-1}$, equation (4.4) admits exactly one solution for every $u \in \mathcal{U}_{T}$. In fact, for $\varepsilon=0$ this follows from the hyperbolicity of $A$, and for $|\varepsilon|<\left(2 K_{A} M_{2}\right)^{-1}$ we have

$$
\begin{gathered}
\left\|\Phi\left(u, x_{1}, \varepsilon\right)-\Phi\left(u, x_{2}, \varepsilon\right)\right\|_{1} \leq|\varepsilon|\left\|(L-\bar{A})^{-1}\right\|\left\|\bar{F}\left(u, x_{1}, \varepsilon\right)-\bar{F}\left(u, x_{2}, \varepsilon\right)\right\|_{0} \\
\leq|\varepsilon| K_{A} M_{2}\left\|x_{1}-x_{2}\right\|_{1} \leq \frac{1}{2}\left\|x_{1}-x_{2}\right\|_{1} .
\end{gathered}
$$

Hence, for $|\varepsilon|<\left(2 K_{A} M_{2}\right)^{-1}$ and every $u \in \mathcal{U}_{T}, \Phi(u, \cdot, \varepsilon)$ is a contraction. Then, the Banach Contraction Theorem yields the existence of a unique fixed point which we denote by $x(\cdot, u, \varepsilon)$. Furthermore, for fixed $T>0, x(\cdot, u, \varepsilon)$ depends continuously on $(u, \varepsilon) \in \mathcal{U}_{T} \times\left[-\varepsilon_{0}, \varepsilon_{0}\right]$ (see e.g. [8], Proposition 1.2).

To prove the last assertion, notice that for a fixed point $x$ of $\Phi(u, \cdot, \varepsilon)$ one has

$$
\begin{aligned}
\|x\|_{1}= & \|\Phi(u, x, \varepsilon)-\Phi(0,0, \varepsilon)\|_{1} \\
& \leq\|\Phi(u, x, \varepsilon)-\Phi(u, 0, \varepsilon)\|_{1}+\|\Phi(u, 0, \varepsilon)-\Phi(0,0, \varepsilon)\|_{1} \\
& \leq \frac{1}{2}\|x\|_{1}+\left\|(L-\bar{A})^{-1}\right\|\left(c_{U}\|B\|+\varepsilon\|\bar{F}(u, 0, \varepsilon)-\bar{F}(0,0, \varepsilon)\|_{0}\right) \\
& \leq \frac{1}{2}\|x\|_{1}+c_{U} K_{A}\left(\|B\|+M_{1}\right) .
\end{aligned}
$$

which implies the inequality (4.2).

This lemma, combined with Proposition 2.3, yields a bound on the control sets with nonvoid interior. 
Corollary 4.2. Let $A, B$ and $F$ be as in Lemma 4.1, and assume that $U$ contains the origin of $\mathbf{R}^{m}$ in its interior and take

$$
\varepsilon_{0}=\min \left\{1, \frac{1}{2 K_{A} M_{2}}, c_{*}\right\}
$$

$c_{*}$ as in Proposition 2.3. Then every control set with nonvoid interior of (1.1) is contained in the closed $2 c_{U} K_{A}\left(\|B\|+M_{1}\right)$-ball of $\mathbf{R}^{d}$ centered at the origin.

Proof. Assume that there exist a point $p$, laying outside the $2 c_{U} K_{A}\left(\|B\|+M_{1}\right)$ ball centered at the origin, but belonging to the interior of a control set. Then, by Proposition 2.3, there exists a periodic solution of (4.1a) whose image contains $p$. This contradicts the inequality (4.2).

The assertion follows since the local accessibility ensures that, for a control set $D$ with nonvoid interior, one has $D \subset \operatorname{cl} i n t D$.

In what follows we denote by $\varphi_{\varepsilon}(\cdot, u, \xi)$ the solution of the Cauchy problem

$$
\left\{\begin{array}{l}
\dot{x}(t)=A x(t)+B u(t)+\varepsilon F(u(t), x(t), \varepsilon), \\
x(0)=\xi
\end{array}\right.
$$

where $u \in \mathcal{U}_{T}, \xi \in \mathbf{R}^{d}$, and $\varepsilon \in \mathbf{R}$ are given.

We want to prove that, reducing $\varepsilon_{0}$ if necessary, given $T>0$ the image of the periodic solution given by the lemma above is contained in the interior of a control set, provided that the pair $(A, B)$ is controllable.

Let $\mathcal{E}$ be the Banach subspace of $C\left(\mathbf{R}, \mathbf{R}^{m}\right)$ given by

$$
\mathcal{E}=\left\{v \in C\left(\mathbf{R}, \mathbf{R}^{m}\right): \operatorname{supp}(v) \subset[0,1]\right\}
$$

Let $\bar{u}$ with $\bar{u}(t) \in \operatorname{int} U$ for all $t \in \mathbf{R}$ be given. Define the open subset $\mathcal{V}_{\bar{u}}$ of $\mathcal{E}$ as follows:

$$
\mathcal{V}_{\bar{u}}=\{v \in \mathcal{E}: \bar{u}(t)+v(t) \in \operatorname{int} U, \text { for all } t \in \mathbf{R}\} .
$$

Given $\varepsilon \in \mathbf{R}$ and $p_{0} \in \mathbf{R}^{d}$ define the map $\Theta_{\varepsilon, p_{0}}: \mathcal{V}_{\bar{u}} \rightarrow \mathbf{R}^{d}$ by

$$
\Theta_{\varepsilon, p_{0}}(v)=\varphi_{\varepsilon}\left(1, \bar{u}+v, p_{0}\right) .
$$

Let $p_{1}=\Theta_{\varepsilon, p_{0}}(0)$, we want to show that, under suitable assumptions on $A, B$ and $F$, for $\varepsilon$ small enough, there exists a neighborhood of $p_{1}$ which consists of images of $\Theta_{\varepsilon, p_{0}}$.

Lemma 4.3. Assume that the pair $(A, B)$ is controllable and that $F$ is $C^{1}$ with $\left\|D_{1} F\right\| \leq M_{1}$ and $\left\|D_{2} F\right\| \leq M_{2}$ uniformly. Then there exists $\varepsilon_{0}>0$ such that for $|\varepsilon| \leq \varepsilon_{0}, p_{0} \in \mathbf{R}^{d}, \bar{u} \in$ int $\mathcal{U}$, there exists a neighborhood $V$ of 0 in $\mathcal{E}$, such that $\Theta_{\varepsilon, p_{0}}(0)=\varphi_{\varepsilon}\left(1, \bar{u}, p_{0}\right)$ lies in the interior of $\Theta_{\varepsilon, p_{0}}(V)$.

Furthermore, one can actually choose $\varepsilon_{0}$ of the form

$$
\varepsilon_{0}=\min \left\{1, \frac{e^{-2\|A\|} r_{A, B}}{M_{1}+M_{2}\left(\|B\|+M_{1}\right) e^{M_{2}}}\right\},
$$

where $r_{A, B}>0$ depends only on $A$ and $B$.

Proof. By the Surjective Mapping Theorem, it is enough to prove that there exists $\varepsilon_{0}>0$, independent of $\bar{u}$ such that $\Theta_{\varepsilon, p_{0}}^{\prime}(0)$ is surjective for all $|\varepsilon|<\varepsilon_{0}$, and $p_{0}$. 
For $\varepsilon=0$ one can write explicitly

$$
\Theta_{0, p_{0}}^{\prime}(0) \omega=\int_{0}^{1} e^{(1-s) A} B \omega(s) d s
$$

Note that $\Theta_{0, p_{0}}^{\prime}(0): \mathcal{E} \rightarrow \mathbf{R}^{d}$ does depend neither on $\bar{u}$ nor on $p_{0}$, and, by the controllability assumption on $(A, B)$, it is surjective. Let us put $\Theta_{0, p_{0}}^{\prime}(0)=\Lambda$.

Since the surjective linear maps form an open subset of the space $L\left(\mathcal{E}, \mathbf{R}^{d}\right)$, we have that there exists $r_{A, B}>0$ such that any $H \in L\left(\mathcal{E}, \mathbf{R}^{d}\right)$, which satisfies $\|H-\Lambda\| \leq r_{A, B}$, is surjective.

Let us consider now $\varepsilon>0$. Observe that $\Theta_{\varepsilon, p_{0}}^{\prime}(0)=D_{2} \varphi\left(1, \bar{u}, p_{0}\right)$. For $\omega \in \mathcal{E}$ we put

$$
\begin{aligned}
\alpha(t) & =D_{2} \varphi_{\varepsilon}\left(t, \bar{u}, p_{0}\right) \omega, \\
\beta(t) & =D_{2} \varphi_{0}\left(t, \bar{u}, p_{0}\right) \omega .
\end{aligned}
$$

We get

$$
\begin{gathered}
\alpha(t)=\int_{0}^{t}\left[A \alpha(s)+B \omega(s)+\varepsilon D_{1} F\left(\bar{u}(s), \varphi_{\varepsilon}\left(s, \bar{u}, p_{0}\right), \varepsilon\right) \omega(s)\right. \\
\left.+\varepsilon D_{2} F\left(\bar{u}(s), \varphi_{\varepsilon}\left(s, \bar{u}, p_{0}\right), \varepsilon\right) \alpha(s)\right] d s
\end{gathered}
$$

and analogously

$$
\beta(t)=\int_{0}^{t}[A \beta(s)+B \omega(s)] d s .
$$

Hence,

$$
|\alpha(t)| \leq\|\omega\|\left(\|B\|+\varepsilon M_{1}\right)+\int_{0}^{t}\left(\varepsilon M_{2}+\|A\|\right)|\alpha(s)| d s
$$

where $M_{1}$ and $M_{2}$ are upper bounds for $\left\|D_{1} F\right\|$ and $\left\|D_{1} F\right\|$ respectively. By the Gronwall inequality, we get the following estimate for $|\alpha|$

$$
|\alpha(t)| \leq\|\omega\|\left(\|B\|+\varepsilon M_{1}\right) e^{t\left(\|A\|+\varepsilon M_{2}\right)} .
$$

Moreover, using (4.7) and (4.8),

$$
\begin{aligned}
|\alpha(t)-\beta(t)| \leq & \varepsilon\left(M_{1}\|\omega\|+\int_{0}^{1} M_{2}|\alpha(s)| d s\right) \\
& +\int_{0}^{t}\|A\||\alpha(s)-\beta(s)| d s .
\end{aligned}
$$

Plugging (4.9) into (4.10), and assuming $\varepsilon \leq 1$, we get

$$
\begin{aligned}
|\alpha(t)-\beta(t)| \leq \varepsilon\|\omega\|\left(M_{1}+M_{2}\left(\|B\|+\varepsilon M_{1}\right) e^{\|A\|+\varepsilon M_{2}}\right) \\
\quad+\int_{0}^{t}\|A\||\alpha(s)-\beta(s)| d s \\
\leq \varepsilon D\|\omega\|+\int_{0}^{t}\|A\||\alpha(s)-\beta(s)| d s,
\end{aligned}
$$

where we have put

$$
D=e^{\|A\|}\left(M_{1}+M_{2}\left(\|B\|+M_{1}\right) e^{M_{2}}\right) .
$$


Note that the estimate (4.11) is independent of $\bar{u}$ and $p_{0}$. Applying the Gronwall inequality to (4.11),

$$
\sup _{t \in[0,1]}|\alpha(t)-\beta(t)| \leq \varepsilon D\|\omega\| e^{\|A\|} .
$$

In other words, recalling the definitions of $\alpha$ and $\beta$, for

$$
\varepsilon \leq \min \left\{1, \frac{e^{-2\|A\|} r_{A, B}}{M_{1}+M_{2}\left(\|B\|+M_{1}\right) e^{M_{2}}}\right\}
$$

we have

$$
\left\|\Theta_{\varepsilon, p_{0}}^{\prime}(0)-\Theta_{0, p_{0}}^{\prime}(0)\right\|=\left\|\Theta_{\varepsilon, p_{0}}^{\prime}(0)-\Lambda\right\| \leq r_{A, B},
$$

independently of $\bar{u}$ and $p_{0}$, which yields the surjectivity of $\Theta_{\varepsilon, p_{0}}^{\prime}(0)$ for each $\bar{u}$.

Remark 4.4. Lemma 4.3 says that, if $\varepsilon$ is small enough, then, given $\bar{u} \in \operatorname{int} \mathcal{U}$, it is possible to reach any point in a suitably small neighborhood of $\varphi_{\varepsilon}\left(1, \bar{u}, p_{0}\right)$ by varying the control function in a neighborhood of $\bar{u}$.

With only minor changes in the proof one can show that the set which can be reached in a given time $\tau \in(0,1]$ from any given point $p_{0}$ has nonempty interior. This property, often called strong accessibility, obviously implies local accessibility. Hence one can actually choose the constant $c_{*}$ which appears in Proposition 2.3 equal to $\varepsilon_{0}$ in (4.6).

We need to extend the result of Lemma 4.3 to the case of a time $T>1$.

For any $v \in \mathcal{E}$, we put $\tilde{v}(t)=v(t-T+1)$ and define

$$
\Psi_{T, \varepsilon, p_{0}}(v)=\varphi_{\varepsilon}\left(T, \tilde{v}, p_{0}\right)=\Theta_{\varepsilon, \varphi_{\varepsilon}\left(T-1, \bar{u}, p_{0}\right)}(v) .
$$

Thus we immediately get

Corollary 4.5. Assume that the pair $(A, B)$ is controllable and that $F$ is $C^{1}$ with $\left\|D_{1} F\right\| \leq M_{1}$ and $\left\|D_{2} F\right\| \leq M_{2}$ uniformly. Then, there exists $\varepsilon_{0}>0$ such that for $|\varepsilon| \leq \varepsilon_{0}, p_{0} \in \mathbf{R}^{d}, \bar{u} \in \mathcal{U}$ and $T>1$ given, there exists a neighborhood $V$ of 0 in $\mathcal{E}$, such that int $V \subset \Psi_{T, \varepsilon, p_{0}}(V)$.

Furthermore, one can actually choose $\varepsilon_{0}$ of the form (4.6).

Proof. It follows from Lemma 4.3 applied to the function $\tilde{\tilde{u}}: t \mapsto \bar{u}(t-T+1)$ and to the point $\Theta_{\varepsilon, \varphi_{\varepsilon}\left(T-1, \bar{u}, p_{0}\right)}\left(p_{0}\right)$.

This corollary allows us to prove for (1.1) a result which is analogous to Lemma 3.10. From now on we will assume that

$$
\varepsilon_{0}=\min \left\{1, \frac{e^{-2\|A\|} r_{A, B}}{M_{1}+M_{2}\left(\|B\|+M_{1}\right) e^{M_{2}}}, \frac{1}{2 K_{A} M_{2}}\right\}
$$

Lemma 4.6. Let $U$ have non empty interior. Assume that $A$ is hyperbolic, that the pair $(A, B)$ is controllable and that $F$ is $C^{1}$ with $\left\|D_{1} F\right\|$ and $\left\|D_{2} F\right\|$ bounded. Then if $|\varepsilon| \leq \varepsilon_{0}$, given $T>0$ and $\bar{u} \in \operatorname{int} \mathcal{U}_{T}$, (4.1a) has a unique $T$-periodic solution. Furthermore this solution is contained in the interior of a control set of (1.1).

Proof. Observe that, given $T>0$, a $T$-periodic function is also $n T$-periodic, $n \in \mathbf{N}$. Hence, without loss of generality, we can assume $T>1$.

Lemma 4.1 yields the existence of a unique $T$-periodic solution of (4.1a) for $|\varepsilon| \leq \varepsilon_{0}$ and $\bar{u} \in \operatorname{int} \mathcal{U}_{T}$. 
Fix $\bar{u} \in \operatorname{int} \mathcal{U}_{T}$, let $p_{0}$ be the starting point of the unique periodic $T$-periodic solution of (4.1a). From Corollary 4.5 it follows that there exists a neighborhood $V$ of $p_{0}$ in $\mathbf{R}^{d}$ such that for any $q \in V$ there exists $w \in \operatorname{int} \mathcal{U}_{T}$ such that $q=$ $\varphi_{\varepsilon}\left(T, w, p_{0}\right)$.

Considering the time reversed system and reducing $V$, if necessary, we can assume that within this set any point can be driven into any other point. Hence $V$ is contained in the interior of a control set. To prove that the whole $\varphi_{\varepsilon}\left([0, T], \bar{u}, p_{0}\right)$ is contained in the interior of a control set, we proceed as in the last part of the proof of Lemma 3.10 .

A noteworthy consequence of Lemma 4.6 combined with Proposition 2.3 is the following.

Remark 4.7. Assume, in addition to the hypotheses of Lemma 4.6, that $U$ contains 0 in its interior and that $F(0,0, \varepsilon) \equiv 0$ for any $|\varepsilon| \leq \varepsilon_{0}$. Then the origin of $\mathbf{R}^{d}$ is contained in the interior of a control set. In fact, the origin can be regarded as a 1-periodic solution of (4.1a).

We are now in a position to prove the main result of this section.

Theorem 4.8. Let $U$ be convex with non empty interior. Assume that $(A, B)$ in (1.1) is controllable and $A$ is hyperbolic. Let $F$ be $C^{1}$ with $\left\|D_{1} F\right\|$ and $\left\|D_{2} F\right\|$ bounded. Then the control process (1.1) admits exactly one control set $D$ with nonvoid interior if $|\varepsilon| \leq \varepsilon_{0}, \varepsilon_{0}$ as in (4.12).

Furthermore,

1. for $|\varepsilon| \leq \varepsilon_{0}$ the control set $D$ is contained in the $2 c_{U} K_{A}\left(\|B\|+M_{1}\right)$-ball of $\mathbf{R}^{d}$ centered at the origin,

2. if $F(0,0, \varepsilon) \equiv 0$ for any $|\varepsilon| \leq \varepsilon_{0}$, then the origin is contained in the interior of $D$.

Proof. Let $T>1$ and $\bar{u} \in \operatorname{int} \mathcal{U}_{T}$. Lemma 4.1 guarantees the existence of a $T$ periodic solution of (4.1a), whose image is, by Corollary 4.5, contained in the interior of a control set. This proves the existence of at least one control set.

Let us prove the uniqueness assertion. Assume by contradiction that for some $\varepsilon \in\left[-\varepsilon_{0}, \varepsilon_{0}\right]$ there exist two different control sets, say $D_{0}$ and $D_{1}$. Then, by Proposition 2.3, there exists $u_{i} \in \operatorname{int} \mathcal{U}_{T_{i}}, i \in\{0,1\}$, such that the corresponding $T_{i}$-periodic trajectory of (4.1a) is contained in the interior of $D_{i}$. As in the proof of Lemma 4.6 we can always assume that $T_{i}>1$ for $i \in\{0,1\}$.

As in the proof of Theorem 3.12, put $T_{\lambda}=\lambda T_{1}+(1-\lambda) T_{0}$ and define

$$
u_{\lambda}(t)=\lambda u_{1}\left(\frac{T_{1} t}{T_{\lambda}}\right)+(1-\lambda) u_{0}\left(\frac{T_{0} t}{T_{\lambda}}\right) .
$$

Since $U$ is assumed convex, $u_{\lambda} \in \operatorname{int} \mathcal{U}_{T_{\lambda}}$. By the choice of $\varepsilon_{0}$, the equation

$$
\dot{x}(t)=A x(t)+B u_{\lambda}(t)+\varepsilon F\left(u_{\lambda}(t), x(t), \varepsilon\right),
$$

admits a unique $T_{\lambda}$-periodic solution whose image is contained in the interior of a control set. By the argument used in the proof of Theorem 3.12 we get the existence of a continuum which joins $D_{0}$ and $D_{1}$ and whose points are all contained in the interior of a control set. This yields the desired contradiction.

The last two assertions follow from Corollary 4.2 and Remark 4.7. 
Theorem 4.8 has the following remarkable consequence.

Corollary 4.9. Assume $(A, B)$ controllable. Let $G: \mathbf{R}^{m} \times \mathbf{R}^{d} \rightarrow \mathbf{R}^{d}$ be a $C^{1}$ function such that $\left\|D_{1} G(v, p)\right\| \leq M_{1}$ and $\left\|D_{2} G(v, p)\right\| \leq M_{2}$, for any $(v, p) \in$ $\mathbf{R}^{m} \times \mathbf{R}^{d}$. If the bounds $M_{1}$ and $M_{2}$ for the partial derivatives are small enough, then the control process (1.3) admits a unique control set D with nonempty interior. Moreover $D$ turns out to be bounded, and, if $G(0,0)=0$, then $D$ contains the origin of $\mathbf{R}^{m}$ in its interior.

Proof. If $M_{1}$ and $M_{2}$ are small enough then $\varepsilon_{0}=1$ in formula (4.12). Hence the assertion follows directly from Theorem 4.8 .

\section{Aknowledgement}

This paper has been written when the second author was visiting Universität Augsburg as a NCN fellow in the TMR program. M. Spadini wishes to thank the Nonlinear Control Network and the personnel of the 'Institut für Mathematik' for their helpful assistance.

\section{REFERENCES}

[1] F. Colonius and W. Kliemann, Limit behavior and genericity for nonlinear control systems, J. Diff. Equations 109 (1994), pp. 8-41.

[2] F. Colonius and W. Kliemann, Continuous, smooth, and control techniques for stochastic dynamics, in Stochastic Dynamics H. Crauel and M. Gundlach eds., Springer-Verlag, 1999.

[3] F. Colonius and W. Kliemann, The Dynamics of Control, Birkhäuser 1999, to appear.

[4] W. Kliemann, Recurrence and invariant measures for degenerate diffusions, Ann. Prob., 15 (1987), pp. 690-707.

[5] S. Lang, Real and Functional Analysis, Graduate Texts in Mathematics 142, Springer-Verlag, New York, 1993.

[6] V. Jurdjevic, Geometric Control Theory Cambridge Studies in Mathematics 51, Cambridge University Press, Cambridge, 1997.

[7] E. Sontag Mathematical Control Theory, 2nd edition, Texts in Applied Mathematics 6, Springer-Velag, New York, 1998.

[8] E. Zeidler, Nonlinear Functional Analysis vol.1, Springer-Verlag, New York, 1986.

Fritz Colonius, Institut für Mathematik, Universität Augsburg, 86135 Augsburg, GERMANY

E-mail address: colonius@math.uni-augsburg.de

Marco Spadini, Dipartimento di Matematica Applicata 'G. Sansone', Via S. Marta 3, I-50139 FIRENZE, ITALY

E-mail address: spadini@alibaba.math.unifi.it 